\title{
Asociación de la enfermedad periodontal con factores de riesgo en adolescentes de 15 años de una institución educativa
}

\author{
Association of periodontal disease with risk factors in adolescents aged 15 years of an educational institution
}

Danitza Geraldine Yataco Barreda ${ }^{1, a}$, Wendy Alejandra Gomez Diaz ${ }^{1, a}$, Milagros Bertha Carrasco Loyola ${ }^{1,2, b, c}$, Francisco Jose Orejuela Ramirez ${ }^{1,3, d, e}$

\section{RESUMEN}

La enfermedad periodontal es una condición inflamatoria que destruye de forma irreversible a los tejidos que soportan al diente, siendo las principales características la profundidad al sondaje, inflamación, edema y eritema. Objetivo: Determinar la asociación de la enfermedad periodontal con factores de riesgo en adolescentes de 15 años de una institución educativa. Material y Métodos: El estudio fue observacional, descriptivo, retrospectivo y transversal basado en los registros de la base de datos del levantamiento epidemiológico de salud bucal del año 2018, contando con una muestra de 29 escolares de 15 años matriculados en el año académico 2018. Las variables fueron enfermedad periodontal, profundidad al sondaje, sangrado gingival, sexo, frecuencia de cepillado, frecuencia de visita al dentista, frecuencia de consumo de tabaco y frecuencia de consumo de alcohol. Para el manejo de los datos se utilizó Excel versión 10 y se exportó a Stata versión 15 en donde se realizaron las pruebas estadísticas. Resultados: La frecuencia de cepillado menor a dos veces al día y el consumo de tabaco se comportaron como factores que aumentan la posibilidad del desarrollo de la enfermedad periodontal con RP: 4,684, IC 95\% (0,406 - 54,002) y RP: 1,468, IC 95\% (0,489 - 4,412), respectivamente; sin embargo, los valores obtenidos no son estadísticamente significativos $(\mathrm{p}>0,05)$. Conclusión: Existe una asociación de la enfermedad periodontal tanto con el cepillado menor de dos veces al día como con el consumo de tabaco en adolescentes de 15 años de una institución educativa.

PALABRAS CLAVE: Enfermedad periodontal, factores de riesgo, adolescentes. 


\section{ABSTRACT}

Periodontal disease is an inflammatory condition that irreversibly destroys the tissues that support the tooth, the main characteristics of this disease being probing depth, inflammation, edema and erythema. Objective: Determine the association of periodontal disease with risk factors in 15-year-old adolescents from an educational institution. Material and Methods: The study was observational, descriptive, retrospective and cross-sectional based on the records of the database of the epidemiological oral health survey of 2018, with a sample of 29 15 -year-old schoolchildren enrolled in the 2018 academic year. The variables were periodontal disease, depth to probing, gingival bleeding, sex, frequency of brushing, frequency of visits to the dentist, frequency of tobacco consumption, and frequency of alcohol consumption. Excel version 10 was used to manage the data and it was exported to Stata version 15 where the statistical tests were performed. Results: The frequency of brushing less than twice a day and the consumption of tobacco would behave as factors that increase the possibility of the development of periodontal disease with RP: 4,684, IC 95\% (0,406 - 54,002) y RP: 1,468, IC 95\% (0,489$4,412)$, respectively; however, the values obtained are not statistically significant ( $p>0.05)$. Conclusion: There is an association of periodontal disease with both brushing less than twice a day and tobacco use in 15-year-old adolescents from an educational institution.

KEY WORDS: Periodontal disease, risk factors, adolescents

\section{INTRODUCCIÓN}

La enfermedad periodontal es una condición inflamatoria que destruye irreversiblemente a los tejidos de soporte del diente y afecta aproximadamente al $20-50 \%$ de la población mundial (1). Al existir una disbiosis de la microbiota en la cavidad oral, da como consecuencia el desarrollo de diversas enfermedades orales, siendo una de ellas la enfermedad periodontal, que se caracteriza por el enrojecimiento de los tejidos gingivales, presencia de placa, sangrado al sondaje y cambios de consistencia y contorno de la encía, influenciando esto en la calidad de vida del paciente $(2,3)$. Podemos apreciar que la mayoría de los países sudamericanos presentan una prevalencia mayor del $15 \%$ de enfermedad periodontal (4) y según los datos epidemiológicos del Ministerio de Salud del Perú los niños entre los 10 y 15 años tienen una prevalencia de periodontitis de $52,5 \%$ (5). Una de las principales causas de la enfermedad periodontal es la inadecuada higiene bucal (6), sin embargo, existen factores que se consideran de riesgo debido a que hacen más susceptibles o predisponen la aparición y progreso de la enfermedad (7).

Es importante la remoción mecánica de la placa dental para evitar el progreso de la enfermedad periodontal, esto se debe a que esta es la principal causa de inflamación. Previos artículos mencionan la importancia del control periodontal tanto por parte de los pacientes, con una buena técnica de cepillado, como por parte de los odontólogos, quienes deben explicar la relevancia de la fase de mantenimiento a los pacientes (8). Se sabe que la importancia de la visita al odontólogo es fundamental para prevenir diversas enfermedades, siendo reflejado esto en el estudio de Brennan et al., donde concluyeron que los pacientes que tienen un mal estado de salud bucal carecen de servicios preventivos y tratamientos oportunos y adecuados (9). Así mismo, el hábito de fumar se considera como otro factor que aumenta la posibilidad del avance de la enfermedad periodontal $(10,11)$. Esto se debe a que impide la correcta función de los neutrófilos y fibroblastos, además que altera la producción de anticuerpos, factores vasculares y mediadores inflamatorios (12).

Se ha evidenciado que los fumadores suelen presentar disminuciones en la respuesta a la terapia periodontal y muestran a lo mucho la mitad de la mejoría luego de distintas intervenciones terapéuticas no quirúrgicas (13). Es importante destacar que la mayoría de las personas con este hábito comienzan desde una edad muy temprana, siendo un mayor porcentaje de consumidores, varones (14), por esta razón es necesaria la intervención temprana para que sea posible prevenir su consumo tan pronto $(15,16)$. El consumo de alcohol es un factor de riesgo para el progreso de la enfermedad periodontal, especialmente en los adolescentes. Esto está evidenciado en el 
estudio realizado por Wiad Lek en el 2019, en donde obtuvieron como resultados que hay una tendencia al aumento de enfermedades precancerosas en función a la duración de adicción a esta sustancia (17).

Para el estudio, se tomó en cuenta los factores que afectan con mayor frecuencia a los adolescentes, los cuales son: frecuencia de cepillado, visita al dentista, consumo de tabaco y consumo de alcohol. Es fundamental realizar estos tipos de estudios epidemiológicos ya que nos permite, de manera global, obtener datos de una población. Existen diversos índices periodontales como alternativas diagnóstico; sin embargo, en nuestro estudio utilizamos el índice periodontal comunitario. La Organización Mundial de la Salud (OMS) recomienda el uso de este índice debido a que sirve como un indicador para la detección de la enfermedad periodontal en una comunidad, lo que nos permitirá planificar conductas sanitarias para la prevención y tratamiento de esta $(18,19,20)$.

El presente estudio tuvo como objetivo determinar la asociación de la enfermedad periodontal con los diferentes factores de riesgo que se han mencionado en adolescentes de 15 años de una institución educativa.

\section{MATERIAL Y MÉTODOS}

El estudio fue de tipo observacional, descriptivo, retrospectivo y transversal. Este fue basado en los registros de escolares evaluados por estudiantes de tercer año de estomatología que realizaron un diagnóstico epidemiológico como parte de un programa de salud bucal en el curso de Odontología Social II, estos registros fueron solicitados al Departamento Académico de Odontología Social del año 2018. Se seleccionaron a los escolares de 15 años que se encontraban matriculados en el año académico 2018 con registros en la base de datos de la Institución Educativa "Libertador San Martín", Independencia. Posteriormente, se procedió con la eliminación de los datos personales de los participantes, contando con una muestra de 29 alumnos.

La variable enfermedad periodontal se determinó tomando en cuenta la profundidad al sondaje y el sangrado gingival, existiendo presencia de enfermedad cuando la profundidad al sondaje medida es mayor o igual a $4 \mathrm{~mm}$ y hay presencia de sangrado gingival. La profundidad al sondaje se determinó al explorar la hendidura gingival con una sonda periodontal y estableciendo su medida, en donde existen 3 categorías: sin bolsa, bolsa de 4-5 $\mathrm{mm}$ y bolsa de $6 \mathrm{~mm}$ a más. Para determinar la presencia o ausencia de sangrado gingival se utilizó una sonda periodontal y se evaluaron todas las piezas dentales presentes en boca de cada alumno. La variable sexo se categorizó en femenino y masculino. La frecuencia de cepillado es una variable con la cual determinamos las veces en las que el alumno se cepilla los dientes y se obtuvo este dato mediante la aplicación de un cuestionario, siendo categorizada en: nunca, una vez por semana, varias veces a la semana (2-6 veces), una vez al día y dos veces al día o más. La visita al dentista es una variable que determinó la frecuencia con la cual el estudiante acude a las visitas odontológicas, se obtuvo este dato aplicando una encuesta cuyas categorías fueron: una vez, dos veces, tres veces o más, ninguna (no he visitado al dentista en los últimos 12 meses), nunca he recibido atención dental o visitado al dentista en toda mi vida y no sé/no recuerdo. La frecuencia de consumo de tabaco y la frecuencia de consumo de alcohol se evaluaron mediante la aplicación de una encuesta y sus categorías fueron: todos los días, algunas veces a la semana, una vez a la semana, algunas veces al mes, pocas veces y nunca.

Para el manejo de los datos se utilizó Excel versión 10, en donde se tuvo la base de datos brindada por el Departamento de Odontología Social y para la confidencialidad de los datos personales, se eliminaron los dichos datos de la base de datos y solo se trabajó por códigos. Posteriormente, se exportó a Stata versión 15 en donde se realizaron las pruebas estadísticas para determinar si existen diferencias significativas. Para el análisis descriptivo de datos se emplearon tablas de distribución de frecuencias absolutas y relativas de las variables cualitativas. Para asociar la enfermedad periodontal y los factores de riesgo se empleó la prueba no paramétrica de Chicuadrado y regresión de Poisson. El estudio se realizó con un nivel de confianza del $95 \%$ y un $\mathrm{p}<0,05$.

El proyecto se ejecutó una vez que se contó con la aprobación del Comité Institucional de Ética de la Universidad Peruana Cayetano Heredia (CIE-UPCH) con fecha 09 de diciembre del 2019 y código SIDISI $\mathrm{N}^{\circ} 200214$. 


\section{RESULTADOS}

Se llevó a cabo un análisis descriptivo con la muestra analítica conformada por 29 alumnos de 15 años. Se evidencia que el 34,48\% $(n=10)$ de los adolescentes presentan enfermedad periodontal, en donde $13,79 \%(\mathrm{n}=4)$ pertenecen al sexo femenino y $44,83 \%(n=6)$, al sexo masculino. El 65,52\% $(n=19)$ de la muestra no presenta enfermedad periodontal, en el cual el $41,38 \%(n=12)$ son del sexo femenino y $24,14 \%(n=7)$, del sexo masculino (tabla 1$)$.
Con respecto a los resultados obtenidos en la encuesta aplicada, se observa que $20.68 \%(n=6)$ de los alumnos han visitado una vez al odontólogo, en donde la mitad de estos no presenta enfermedad periodontal. El 79,31\% (n=23) de los alumnos se cepillan dos veces al día o más, en donde el 48,28\% $(\mathrm{n}=14)$ no presentan enfermedad periodontal. Asimismo, con respecto al consumo de tabaco, se destaca que el $86,21 \%(n=25)$ nunca lo consumió y el 31,03\% $(n=9)$ de estos, presentan enfermedad periodontal. Según los resultados obtenidos, el $75,86 \%$ de los alumnos nunca consumieron alcohol (tabla 1).

Tabla 1. Asociación de la enfermedad periodontal con sexo, frecuencia de visita al dentista, frecuencia de cepillado, consumo de tabaco y consumo de alcohol en adolescentes de 15 años matriculados en el año académico 2018 en una institución educativa.

\begin{tabular}{|c|c|c|c|c|c|}
\hline & & \multicolumn{3}{|c|}{ Enfermedad Periodontal } & \multirow[b]{2}{*}{ Valor $\mathbf{p}$} \\
\hline & & No presenta & Presenta & Total & \\
\hline \multicolumn{6}{|l|}{ Sexo } \\
\hline & Femenino & $12(41,38)$ & $4(13,79)$ & $16(55,17)$ & \multirow{2}{*}{0,233} \\
\hline & Masculino & $7(24,14)$ & $6(20,69)$ & $13(44,83)$ & \\
\hline \multicolumn{6}{|c|}{ Frecuencia de visita al dentista } \\
\hline & Una vez & $3(10,34)$ & $3(10,34)$ & $6(20,69)$ & \multirow{6}{*}{0,287} \\
\hline & Dos veces & $0(0,00)$ & $1(3,45)$ & $1(3,45)$ & \\
\hline & Tres veces o más & $2(6,90)$ & $3(10,34)$ & $5(17,24)$ & \\
\hline & $\begin{array}{l}\text { Ninguna, no he visitado al dentista en } \\
\text { los últimos } 12 \text { meses }\end{array}$ & $7(24,14)$ & $2(6,90)$ & $9(31,03)$ & \\
\hline & $\begin{array}{l}\text { Nunca he recibido atención dental o } \\
\text { visitado al dentista en toda mi vida }\end{array}$ & $3(10,34)$ & $1(3,45)$ & $4(13,79)$ & \\
\hline & No sé / No recuerdo & $4(13,79)$ & $0(0,00)$ & $4(13,79)$ & \\
\hline \multicolumn{6}{|c|}{ Frecuencia de cepillado } \\
\hline & Una vez al día & $4(13,79)$ & $1(3,45)$ & $5(17,24)$ & \multirow{3}{*}{0,418} \\
\hline & Dos veces al día o más & $14(48,28)$ & $9(31,03)$ & $23(79,31)$ & \\
\hline & No respodende & $1(3,45)$ & $0(0,00)$ & $1(3,45)$ & \\
\hline \multicolumn{6}{|c|}{ Frecuencia de consumo de cigarrillos } \\
\hline & Nunca & $16(55,17)$ & $9(31,03)$ & $25(86,21)$ & \multirow{4}{*}{0,693} \\
\hline & Solo una vez & $1(3,45)$ & $1(3,45)$ & $2(6,90)$ & \\
\hline & Varias veces al mes & $1(3,45)$ & $0(0,00)$ & $1(3,45)$ & \\
\hline & No Responde & $1(3,45)$ & $0(0,00)$ & $1(3,45)$ & \\
\hline \multicolumn{6}{|c|}{ Frecuencia de consumo de alcohol } \\
\hline & Nunca & $13(44,83)$ & $9(31,03)$ & $22(75,86)$ & \multirow{4}{*}{0,123} \\
\hline & Solo una vez & $4(13,79)$ & $0(0,00)$ & $4(13,79)$ & \\
\hline & Varias veces al mes & $0(0,00)$ & $1(3,45)$ & $1(3,45)$ & \\
\hline & No Responde & $2(6,90)$ & $0(0,00)$ & $2(6,90)$ & \\
\hline Total & & $19(65,52)$ & $10(34,48)$ & $29(100,0)$ & \\
\hline
\end{tabular}


Tabla 2. Factores de riesgo asociados a enfermedad periodontal en adolescentes

\begin{tabular}{|c|c|c|c|c|c|c|}
\hline Factor de riesgo & RP crudo* & Valor $p$ & Intervalo Confianza 95\% & RP ajustado** & Valor $\mathbf{p}$ & Intervalo Confianza 95\% \\
\hline Sexo & 0,5416 & 0,253 & {$[0,1894994-1,548304]$} & 0,3102 & 0,01 & {$[0,1279555-0,7521838]$} \\
\hline $\begin{array}{l}\text { Frecuencia de } \\
\text { cepillado } \\
\text { Menor de dos veces } \\
\text { al día }\end{array}$ & 1,9565 & 0,479 & {$[0,3048375-12,55744]$} & 4,6840 & 0,216 & {$[0,4062774-54,00228]$} \\
\hline $\begin{array}{l}\text { Frecuencia visita al } \\
\text { dentista } \\
\text { No ha ido en el último } \\
\text { año }\end{array}$ & 0,3956 & 0,105 & {$[0,1288088-1,21494]$} & 0,2873 & 0,004 & {$[0,1229116-0,67154]$} \\
\hline $\begin{array}{l}\text { Frecuencia de } \\
\text { consumo de } \\
\text { cigarrillos } \\
\text { Alguna vez en su vida }\end{array}$ & 0,9259 & 0,93 & {$[0,1667358-5,1419]$} & 1,4688 & 0,493 & {$[0,4889774-4,412174]$} \\
\hline $\begin{array}{l}\text { Frecuencia de } \\
\text { consumo de alcohol } \\
\text { Alguna vez en su vida }\end{array}$ & 0,4889 & 0,45 & {$[0,076236-3,135163]$} & 0,7301 & 0,799 & {$[0,0648005-8,225857]$} \\
\hline
\end{tabular}

* Regresión de Poisson simple con varianza robusta

** Regresión de Poisson múltiple con varianza robusta

Por último, se puede observar que la frecuencia de cepillado menor de dos veces al día y el consumo de tabaco son factores que aumentan la posibilidad del desarrollo de la enfermedad periodontal con valores de RP: 4,684, IC 95\% (0,406 - 54,002) y RP: 1,468, IC 95\% (0,489 - 4,412), respectivamente. Se debe mencionar que los resultados no son estadísticamente significativos $(p>0,05)$ (tabla 2$)$.

\section{DISCUSIÓN}

La prevalencia de enfermedad periodontal encontrada fue de $34.48 \%$ en los adolescentes de 15 años de la institución educativa. De igual forma, en el estudio realizado por Das et al., publicado el 2017 se puede ver que la enfermedad periodontal estaba presente en el $75 \%$ de la muestra evaluada (21), se debe tener presente que, en el estudio realizado, la muestra solo consideraba alumnos de 15 años, a diferencia del estudio citado que incluyen alumnos de 15 a 17 años.

Los hombres tuvieron una mayor prevalencia de la enfermedad periodontal que las mujeres, en una relación de 3 a 2 . De igual manera se encontró el mismo resultado en la revisión sistemática que se llevó a cabo en adolescentes de China realizado por Xiao et al., y diversos estudios $(21,22,23)$. Similarmente, Khan et al., mencionan que esto sucede debido a que los varones se encuentran más expuestos a diversos factores de riesgo, tales como un cepillado dental deficiente y el inicio temprano del consumo de tabaco (14).

Das et al., determinaron que a pesar de que los adolescentes tienen prácticas de higiene bucal favorables, sus técnicas utilizadas muchas veces no son adecuadas, lo que da como resultado una alta prevalencia de enfermedad periodontal (21). Este mismo resultado se evidenció en nuestro estudio, ya que el 79,31\% de los adolescentes mencionan que cepillan sus dientes dos o más veces al día, teniendo una prevalencia de enfermedad periodontal de $34.48 \%$. Según los datos obtenidos, los que se cepillan 1 vez o menos al día, la relación de no presentar enfermedad y presentarla es de $4: 1$, pero en los que se cepillan 2 veces o más es de 14:9, correspondientemente. En el análisis de regresión de Poisson se verifica que cepillarse una vez o menos veces al día es un factor que aumenta la posibilidad de sufrir enfermedad periodontal $(p=0,216)$. Con estos resultados se puede mencionar que al igual que Olczak-kowalczyk et al., la mala higiene bucal es un predictor de la enfermedad periodontal (23). Con esta primicia, se debe considerar promover la salud dental para mejorar las prácticas de higiene (24). Además, debe existir un control de la técnica de cepillado (8), para verificar la adecuada eliminación de la 
placa bacteriana, sobre todo en la zona interdental ya que Amanda Gallie en el 2019 (24) menciona la importancia de implementarla para así mejorar los resultados de un cepillado (24).

Se sabe que el consumo de tabaco es un factor de riesgo ambiental para la enfermedad periodontal, debido a que el humo inhalado produce una vasoconstricción en los tejidos periodontales produciendo exacerbación de la progresión de la enfermedad periodontal (25). Sin embargo, el 86,21\% de los adolescentes evaluados en nuestro estudio nunca había consumido cigarrillos en su vida. En la tabla 2 se puede ver que consumir cigarrillos aumenta la probabilidad de tener enfermedad periodontal $(p=0,493)$. Esto se confirmar con el estudio realizado por Nociti et al., en donde mencionan que los fumadores tienen una mayor susceptibilidad a la rápida progresión de la enfermedad periodontal (26). De la misma forma, Khan et al., en su estudio con un total de 443 participantes evaluados y entrevistados sobre sus hábitos orales, encuentra que fumar en exceso conduce a un mayor riesgo de tener periodontitis (14). Por último, Ramseier et al., mencionan que dejar de fumar antes de los 30 años es importante para prevenir la pérdida temprana de dientes con el fin de asegurar una dentición mayor a 20 dientes a los 60 años (27).

Así como el consumo de tabaco es un factor de riesgo ambiental, el consumo de alcohol también lo es (25). Sankaranarayanan et al., refieren que para evaluar la asociación del consumo de alcohol con la salud periodontal se debe tener en cuenta diferentes características tales como la edad, el género y posición socioeconómica (28). Skrypnikov et al.,. evaluaron la condición de tejidos periodontales y mucosa oral en pacientes hospitalizados de entre 14 y 17 años, quienes habían abusado del alcohol durante dos y tres años, encontrando que el alcohol tiene un efecto adverso en la salud periodontal (17). Sin embargo, en nuestro estudio el consumo de alcohol no fue considerado un factor asociado a la enfermedad periodontal ya que el 75,86\% (n=22) de los evaluados, nunca lo había consumido.

En el estudio se puede evidenciar que el 31,03\% de los alumnos nunca han visitado al odontólogo. Se sabe que la frecuencia de visita al dentista funciona como un factor protector puesto que es una forma de prevenir el progreso de diversas enfermedades bucales. Con el reporte de Brennan et al., en donde determinan que la carencia de servicios preventivos y tratamientos oportunos y apropiados es un factor de riesgo para la presencia de enfermedad periodontal (9), se puede evidenciar la importancia de la visita al odontólogo para evitar el progreso de dicha enfermedad.

Finalmente, con el análisis realizado a las variables, se debe mencionar que los resultados obtenidos en el estudio no son estadísticamente significativos $(\mathrm{p}>0,05)$. Esto se debe a la limitación del tamaño reducido de la muestra $(n=29)$, dado que genera una imprecisión en la estimación realizada e imposibilita generalizar los resultados a toda la población.

Fueimportanterealizar este estudio epidemiológico ya que con los resultados obtenidos, se reafirma y se contribuye con mayor evidencia científica que la frecuencia de cepillado y el consumo de tabaco son factores que aumentan la posibilidad de desarrollar enfermedad periodontal en adolescentes.

\section{CONCLUSIONES}

Se puede concluir de manera general que existe asociación de la enfermedad periodontal con los factores de riesgo en adolescentes de 15 años. La frecuencia de cepillado menor de dos veces al día y el consumo de tabaco son factores que aumentan la posibilidad de desarrollar la enfermedad periodontal. Sin embargo, estos resultados no son estadísticamente significativos, debido a que los resultados obtenidos en el estudio no son concluyentes puesto que nuestra evaluada fue muy reducida.

Conflicto de intereses: los autores no tienen conflicto de interés con este informe.

Aprobación de ética: Aprobado (Constancia 699-2919).

Financiamiento: Ninguno.

Contribuciones de los autores: todos los autores contribuyeron a este manuscrito. 


\section{Correspondencia:}

\section{Danitza Geraldine Yataco Barreda}

Rua Professor Vieira de Almeida 5, 5G, 1600-664

Lisboa, Portugal

Teléfono: +351936404077

Correo electrónico: danitza.yataco.b@upch.pe

\section{REFERENCIAS BIBLIOGRÁFICAS}

1. Nazir MA. Prevalence of periodontal disease, its association with systemic diseases and prevention. Int. J. Health Sci (Qassim). 2017; 11: 72-80.

2. Durham J, Fraser HM, McCracken GI, Stone KM, John MT, Preshaw PM. Impact of periodontitis on oral health-related quality of life. J Dent. 2013;41(4):370-6.

3. Lourenco TG, Heller D, Silva-Boghossian CM, Cotton SL, Paster BJ, Colombo AP. Microbial signature profiles of periodontally healthy and diseased patients. J. Clin. Periodontol. 2014; 41: 1027-36.

4. Federación Dental Internacional. El Desafío de las Enfermedades Bucodentales - Una llamada a la acción global. Atlas de Salud Bucodental. 2a ed. Ginebra: Federación Dental Internacional; 2015.

5. Centro Nacional de Epidemiología, Prevención y Control de Enfermedades. Perfil Epidemiologico de Salud Bucal en escolares de 3 a 15 años, Perú 2012 2014. Lima: Ministerio de Salud.

6. De Oliveira C, Watt R, Hamer M. Toothbrushing, inflammation, and risk of cardiovascular disease: Results from Scottish Health Survey. BMJ. 2010; 340:c2451.

7. Casanova L, Hughes FJ, Preshaw PM. Diabetes and periodontal disease: A two-way relationship. Br Dent J. 2014; $217: 433-7$

8. Sánchez S, Martinez B. Terapéutica periodontal de mantenimiento. Av Periodon Implantol. 2017; 29(1):1121.

9. Brennan DS, Roberts-Thomson KF, Spencer AJ. Oral health of Indigenous adult public dental patients in Australia. Aust Dent J. 2007;52(4):322-8.

10. Carvajal P, Gómez M, Gomes S, et al. Prevalence, severity, and risk indicators of gingival inflammation in a multi-center study on South American adults: a cross sectional study. J Appl Oral Sci. 2016; 24(5):52434.

11. Rozier RG, White BA, Slade GD. Trends in Oral Diseases in the U.S. Population. J Dent Educ. 2017;81(8): eS97-109.

12. Khan S, Saub R, Vaithilingam RD, Safii SH, Vethakkan SR, Baharuddin NA. Prevalence of chronic periodontitis in an obese population: a preliminary study. BMC Oral Health. 2015; 15:114.

13. Johnson GK, Hill M. Cigarette smoking and the periodontal patient. J Periodontol. 2004; 75 (2):196209.

14. Khan S, Khalid T, Awan KH. Chronic periodontitis and smoking. Prevalence and dose-response relationship. Saudi Med J. 2016;37(8):889-94.

15. Jashinsky JM, Liles S, Schmitz K, Ding D, Hovell M. Risk factors for tobacco susceptibility in an orthodontic population: An exploratory study. Am J Orthod Dentofac Orthop Off Publ Am Assoc Orthod Its Const Soc Am Board Orthod. 2017;152(2):171-7.

16. Albandar JM, Streckfus CF, Adesanya MR, Winn DM. Cigar, pipe, and cigarette smoking as risk factors for periodontal disease and tooth loss. J Periodontol. 2000; 71(12):1874-1881.

17. Skrypnikov AN, Sheshukova OV, Kazakov OA, Trufanova VP, Polishchuk TV, Tkachenko IM. Oral status in adolescents with alcohol addiction. Wiad Lek. 2019; 72(5):970-971.

18. Su CW, Yen AF, Lai H, Lee Y, Chen HH, Chen, SS. Effects of risk factors on periodontal disease defined by calibrated community periodontal index and loss of attachment scores. Oral Dis. 2017; 23(7):949-955.

19. World Health Organization. Assessment of oral health status. Oral health surveys: basic methods. 5th ed. Brazil: WHO Library Cataloguing in Publication Data; 2013.p. 35-56.

20. Pawlaczyk-Kamieńska T, Torlińska-Walkowiak N, Borysewicz-Lewicka M. The relationship between oral hygiene level and gingivitis in children. Adv Clin Exp Med. 2018; 27(10):1397-1401.

21. Das UM, Vadakkekuttical RJ, Kanakkath H, Shankunni SP. Dental health awareness, attitude, and dental health- care seeking practices as risk indicators for the prevalence of periodontal disease among 15-17-yearold school children in Kozhikode district, Kerala, J Indian Soc of Periodontol. 2017; 21(2):144-151.

22. Xiao L, Karapen K, Dong S, Yang H, Zhang X. Epidemiology of periodontal disease in adolescents in mainland China, 1983-2020: a systematic review and meta-analysis. Ann Palliat Med. 2021; 10(1):4560.

23. Olczak-Kowalczyk D, Gozdowski D, Kaczmarek U. Oral Health in Polish fifteen-year-old adolescents. Oral Health Prev Dent. 2019; 17(2):139-146.

24. Gallie A. Home use of interdental cleaning devices and toothbrushing and their role in disease prevention. Evid Based Dent. 2019; 20 (4):103-104.

25. Chapple ILC, Mealey BL, Van Dyke TE, et al. Periodontal health and gingival diseases and conditions on an intact and a reduced periodontium: consensus report of workgroup 1 of the 2017 World Workshop on the Classification of Periodontal and PeriImplant Diseases and Conditions. J Clin Periodontol. 2018;45:S68-S77.

26. Nociti FH, Casati MZ, Duarte PM. Current perspective 
of the impact of smoking on the progression and treatment of periodontitis. Periodontology 2000; 2014; 67(1):187-210.

27. Ramseier C, Anerud A, Dulac M, et al. Natural history of periodontitis: Disease progression and tooth loss over 40 years. J Clin Periodontol. 2017; 44(12):11821191.
28. Sankaranarayanan R, Keränen AL, Saxlin T, et al. Association between alcohol use and periodontal pockets in Finnish adult population. Acta Odontol Scand. 2019; 77 (5):371-379.

Recibido : 21-05-2021

Aceptado : 28-05-2021 